\title{
Towards efficient and accurate $a b$ initio solutions to periodic systems via transcorrelation and coupled cluster theory
}

\author{
Ke Liao ๑," Thomas Schraivogel $\odot$, Hongjun Luo $\odot$, and Daniel Kats $\odot^{\dagger}$ \\ Max Planck Institute for Solid State Research, Heisenbergstrasse 1, 70569 Stuttgart, Germany \\ Ali Alavi $\odot^{*}$ \\ Max Planck Institute for Solid State Research, Heisenbergstrasse 1, 70569 Stuttgart, Germany \\ and Yusuf Hamied Department of Chemistry, University of Cambridge, Lensfield Road, Cambridge CB2 1EW, United Kingdom
}

(Received 12 March 2021; accepted 29 June 2021; published 20 July 2021)

\begin{abstract}
We propose a streamlined combination of the transcorrelation (TC) and coupled cluster (CC) theory, which not only increases the convergence rate with respect to the basis set but also extends the applicability of the lowestorder CC approximations to strongly correlated systems. Using the three-dimensional uniform electron gas as a model for real periodic solids and highly accurate ground-state energies from the state-of-the-art quantum Monte Carlo methods as benchmark, we show that, with the correct physical insights incorporated, our TC-CC methods gain drastic improvements in accuracy compared with canonical CC methods, and the errors of our methods remain \pm 0.001 Hartree per electron (Ha/electron) across a wide range of densities. With the greatly improved efficiencies and the balanced and accurate performances at both low and high densities, our methods present great promise for applications on real solids with weak and strong correlations.
\end{abstract}

DOI: 10.1103/PhysRevResearch.3.033072

\section{INTRODUCTION}

The coupled cluster (CC) methodologies [1-3] at the level of singles and doubles (CCSD) and perturbative triples $(\mathrm{CCSD}(\mathrm{T}))$ [4] have become the de facto standard of singlereference $a b$ initio quantum chemistry and can be applied to systems consisting of hundreds of electrons [5-8]. In the past few years, these methods have also shown promise in applications to the solid state [9-14], although significant challenges remain before they can be routinely applied, as, for example, density functional theories are. On the one hand, because of quite steep computational scaling $\left(\mathcal{O}\left(N^{6}\right)\right.$ and $\mathcal{O}\left(N^{7}\right)$ for CCSD and $\operatorname{CCSD}(\mathrm{T})$, respectively), it is desirable to keep the methods at the lowest possible CC level, namely, CCSD, while maintaining accuracy. The more accurate $\operatorname{CCSD}(\mathrm{T})$, as a perturbative correction to CCSD, additionally fails for metals [15]. It is also desirable that the CC methods can be extended to more strongly correlated systems, where the single reference nature of these approximations breaks down. There have been various attempts to develop modified CCSD methods with a higher accuracy for weakly [16-19] and

\footnotetext{
*ke.liao.whu@gmail.com

†.kats@fkf.mpg.de

‡a.alavi@fkf.mpg.de
}

Published by the American Physical Society under the terms of the Creative Commons Attribution 4.0 International license. Further distribution of this work must maintain attribution to the author(s) and the published article's title, journal citation, and DOI. Open access publication funded by the Max Planck Society. strongly [20-24] correlated systems. The distinguishable cluster singles and doubles (DCSD) $[25,26]$ is one such method, which has shown promise in improving CCSD in weakly and strongly correlated molecular systems [27-29].

In a separate development, there has been renewed interest in so-called transcorrelated (TC) methods [30-44], based on Jastrow factorization of the electronic wave function, which result in effective similarity-transformed (ST) Hamiltonians [40,43]. Although TC methods were originally proposed as a way to accelerate basis set convergence in electronic wave functions, it has become apparent that such similarity transformations can also be extremely helpful in the context of strongly correlated systems. For example, in the repulsive two-dimensional (2D) Fermi-Hubbard model, it was found that, with a suitable Gutzwiller correlator, extremely compact forms of ground-state right eigenvectors of the ST Hubbard Hamiltonian could be obtained [42], dominated by the Hartree-Fock (HF) determinant. Since single-reference CC methods work best when the wave function is dominated by the HF determinant, and furthermore, since the $\mathrm{CC}$ method can itself be cast in terms of a similarity transformation of the Hamiltonian, it is natural to ask if the two concepts- CC and TC - can be usefully combined into a single framework, whereby the compactification generated by the TC method is exploited by the $\mathrm{CC}$ method to extend its range of applicability into more strongly correlated systems. The purpose of this paper is to report such an investigation, applied to the uniform electron gas (UEG) over a broad range of densities to assess the accuracy of our new methods at different correlation strengths. In particular, we show that a combination of the TC method with the DCD method yields highly accurate energies over the physically relevant range of densities from 
$r_{s}=0.5-10$, implying that this method has potential for applications to real solids.

The 3D UEG model assumes that the background is evenly and positively charged, and that the electrons interact with each other via the Coulomb interaction. As simple as it is, the UEG possesses an intricate phase diagram [45,46], which can only be accurately described by theories that perform consistently well over a broad range of densities. Historically, the UEG model has also played an important role in the development of many useful approximations. For example, several successful local and gradient-corrected density functionals [47-49] are based on the UEG; the random-phase approximation (RPA) [50,51] was developed in a pursuit of understanding metals using the UEG as a model. In recent years, the UEG has attracted studies from various highly accurate $a b$ initio methods and spurred the development of several new methods [15,35,40,52-58].

When applying CC to the UEG, we work in a plane-wave basis; momentum conservation then excludes all single excitations from the CC Ansätz, greatly simplifying the resulting amplitude equations. As a result, the TC Hamiltonian can be treated with relative ease, allowing us to investigate whether the CC method can be beneficially applied to the TC Hamiltonian. We will investigate the CCD and DCD approximations, in the context of the TC Hamiltonian, and show that with a suitable form of the correlator, the basis set convergence can be greatly accelerated (as expected), but in addition, highly accurate energies can be obtained across a broad range of densities $0.5 \leqslant r_{s} \leqslant 50$ covering both the weakly and the strongly correlated regimes. This gives us confidence that the method, once suitably generalized to real systems (which will need to include the singles contribution), will allow a highly accurate yet efficient methodology for the solid state.

In the rest part of this paper, we review the UEG model, $\mathrm{CC}$ and TC theories in Secs. II A, II B, and II C, respectively; in Sec. II D, we discuss the important approximations made to the TC Hamiltonian; we demonstrate our scheme for choosing the optimal parameters in the correlator in Sec. III; we showcase and discuss our TC-CCD/DCD results in comparison with benchmark data in Sec. IV; and finally, we conclude the paper in Sec. V with some outlooks for future directions.

\section{THEORY}

\section{A. 3D UEG}

The 3D UEG is the simplest model for realistic periodic solids, of which the Hamiltonian in real space reads

$$
\hat{H}=-\sum_{i} \frac{1}{2} \nabla_{i}^{2}+\sum_{i \neq j} \frac{1}{\left|\mathbf{r}_{\mathbf{i}}-\mathbf{r}_{\mathbf{j}}\right|}+\text { const. }
$$

where the const. includes the interactions between electrons and the homogeneous positive background charge and the interactions between the electrons and their own periodic images, which is termed as the Madelung constant and will disappear as the size of the simulation cell goes to infinity. Atomic units are used to simplify the equations. When planewave basis functions and a simple cubic simulation cell of volume $\Omega=L^{3}$ are used, we can reformulate the Hamiltonian in a second-quantized form,

$$
\hat{H}=\sum_{p} \sum_{\sigma} \frac{1}{2} \mathbf{k}_{p}^{2} a_{p, \sigma}^{\dagger} a_{p, \sigma}+\frac{1}{2} \sum_{p q r s} \sum_{\sigma \sigma^{\prime}} V_{p q}^{r s} a_{p, \sigma}^{\dagger} a_{q, \sigma^{\prime}}^{\dagger} a_{s, \sigma^{\prime}} a_{r, \sigma},
$$

where for simplicity we use $p, q, r, s \ldots$ indices as a compact form for the general momentum (plane-wave basis function) indices $\mathbf{k}_{p}, \mathbf{k}_{q}, \mathbf{k}_{r}, \mathbf{k}_{s} \ldots$ and hereon we use the two terms plane-wave basis function and orbital equivalently. We stress that due to momentum conservation, i.e., $\mathbf{k} \equiv \mathbf{k}_{r}-\mathbf{k}_{p}=\mathbf{k}_{q}-$ $\mathbf{k}_{s}$, there are only three free indices among pqrs, and the interactions with the homogeneous positive background charge are canceled by the divergent Coulomb potential at $k=0$, which is defined as $V_{p q}^{r s} \equiv V(\mathbf{k})=\frac{4 \pi}{\Omega \mathbf{k}^{2}}$. We also ignore the Madelung contribution in the Hamiltonian which can be added posteriorly to the ground-state energy. The electron density of the system can be described by the Wigner-Seitz radius $r_{s}=$ $\left(\frac{3}{4 \pi N}\right)^{1 / 3} L$, where $N$ is the number of electrons.

\section{B. Coupled/distinguishable cluster doubles}

In the CC Ansätz, we let the many-electron ground-state wave function to be

$$
\Phi=e^{\hat{T}} \Phi_{0},
$$

where $\Phi_{0}$ is the HF wave function and $\hat{T}$ is a cluster operator. In the case of the UEG, we work in a plane-wave basis and $\Phi_{0}$ is given by the Fermi sphere. We will investigate the CCD approximation and its distinguishable variant (DCD) [25,59], which is based on a modification of the CCD amplitude equations by neglecting intercluster exchange diagrams and ensuring the particle-hole symmetry and exactness for two electrons. Alternatively, DCD can be derived from screened Coulomb considerations [60]. We start with the canonical CCD and later highlight the differences between CCD and DCD.

In $\mathrm{CCD}$, the full cluster operator is approximated by the doubles excitations only,

$$
\hat{T} \approx \hat{T}_{2}=\frac{1}{2} \sum_{i j a b} T_{a b}^{i j} \sum_{\sigma \sigma^{\prime}} a_{a, \sigma}^{\dagger} a_{b, \sigma^{\prime}}^{\dagger} a_{j, \sigma^{\prime}} a_{i, \sigma},
$$

where $T_{a b}^{i j}$ are the doubles amplitudes. Following convention, we use $i, j, k \ldots$ and $a, b, c \ldots$ to represent occupied and unoccupied orbitals in the reference determinant, respectively. Again, the momentum conservation ensures that only 3 indices of the amplitude tensor are free, saving a great deal in storing them in the computer memory.

The $T_{2}$ amplitudes are obtained by solving the projective doubles amplitude equations,

$$
\left\langle\Phi_{i j}^{a b}\left|e^{-\hat{T}_{2}} \hat{H} e^{\hat{T}_{2}}\right| \Phi_{0}\right\rangle=0,
$$

where $\Phi_{i j}^{a b}$ are doubly substituted determinants. To be specific, a functional form of the residual, which unifies CCD and DCD for closed-shell systems, can be written as

$$
\begin{aligned}
R_{a b}^{i j}= & V_{a b}^{i j}+V_{a b}^{c d} T_{c d}^{i j}+I_{k l}^{i j} T_{a b}^{k l}+X_{a l}^{c j} T_{c b}^{i l}+\tilde{T}_{a c}^{i k} V_{k l}^{c d} \tilde{T}_{d b}^{l j} \\
& +\hat{\mathcal{P}}(i a ; j b)\left[x_{a}^{c} T_{c b}^{i j}-x_{k}^{i} T_{a b}^{k j}+\chi_{a l}^{c i}\left(T_{b c}^{l j}-T_{c b}^{l j}\right)\right. \\
& \left.-V_{k a}^{i c} T_{c b}^{k j}-V_{k b}^{i c} T_{a c}^{k j}+\tilde{T}_{a c}^{i k} V_{k b}^{c j}\right],
\end{aligned}
$$


where we define the permutation operator $\hat{\mathcal{P}}(i a ; j b) T_{a b}^{i j} \equiv$ $T_{a b}^{i j}+T_{b a}^{j i}$ and the following intermediates,

$$
\begin{aligned}
I_{k l}^{i j} & = \begin{cases}V_{k l}^{i j}+V_{k l}^{c d} T_{c d}^{i j}, & \mathrm{CCD} \\
V_{k l}^{i j}, & \mathrm{DCD}\end{cases} \\
X_{a l}^{c j} & = \begin{cases}V_{k l}^{c d} T_{a d}^{k j}, & \mathrm{CCD} \\
0, & \mathrm{DCD}\end{cases} \\
x_{a}^{c} & = \begin{cases}f_{a}^{c}-\tilde{T}_{a d}^{k l} V_{l k}^{d c}, & \mathrm{CCD} \\
f_{a}^{c}-\frac{1}{2} \tilde{T}_{a d}^{k l} V_{l k}^{d c}, & \mathrm{DCD}\end{cases} \\
x_{k}^{i} & = \begin{cases}f_{k}^{i}+\tilde{T}_{c d}^{i l} V_{l k}^{d c}, & \mathrm{CCD} \\
f_{k}^{i}+\frac{1}{2} \tilde{T}_{c d}^{l l} V_{l k}^{d c}, & \mathrm{DCD}\end{cases} \\
\chi_{a l}^{c i} & = \begin{cases}V_{k l}^{c d} T_{d a}^{k i}, & \mathrm{CCD} \\
0, & \mathrm{DCD}\end{cases} \\
\tilde{T}_{a b}^{i j} & =2 T_{a b}^{i j}-T_{b a}^{i j} .
\end{aligned}
$$

We note that in this case, the Fock matrix $f_{q}^{p}$ is diagonal, with the diagonal elements being the orbital energies $\epsilon_{p}$. A straightforward way to update the $T_{2}$ amplitudes at iteration $n+1$ will be

$$
\begin{aligned}
\Delta_{a b}^{i j} & =\frac{R_{a b}^{i j}}{\epsilon_{i}+\epsilon_{j}-\epsilon_{a}-\epsilon_{b}}, \\
T_{a b}^{i j}(n+1) & =T_{a b}^{i j}(n)+\Delta_{a b}^{i j} .
\end{aligned}
$$

Of course, more advanced iterative schemes can be used, e.g., DIIS [61,62], to accelerate convergence rate.

Using the converged $T_{2}$ amplitudes, the correlation energy is expressed as

$$
E_{\mathrm{c}}=\tilde{T}_{a b}^{i j} V_{i j}^{a b},
$$

and the total energy is

$$
E=E_{\mathrm{HF}}+E_{\mathrm{c}},
$$

where $E_{\mathrm{HF}}=\left\langle\Phi_{0}|\hat{H}| \Phi_{0}\right\rangle$.

\section{TC}

In the TC framework the many-electron wave function is written as

$$
\Psi=e^{\hat{\imath}} \Phi,
$$

where $\hat{\tau}=\frac{1}{2} \sum_{i \neq j} u\left(\mathbf{r}_{\mathbf{i}}, \mathbf{r}_{\mathbf{j}}\right)$ is a correlator consisting of pair correlations $u\left(\mathbf{r}_{\mathbf{i}}, \mathbf{r}_{\mathbf{j}}\right)$, whose form will be discussed later. The $\Phi$ should satisfy the similarity-transformed eigenvalue equation

$$
\hat{H}_{\mathrm{tc}} \Phi=E \Phi, \quad \hat{H}_{\mathrm{tc}}=e^{-\hat{\tau}} \hat{H} e^{\hat{\tau}} .
$$

It is worth pointing out that at this stage, no approximations have been made, and the spectra $E$ of $\hat{H}_{\mathrm{tc}}$ are the same as of the original Hamiltonian. As shown in Ref. [40], the second quantized form of the $\hat{H}_{\mathrm{tc}}$ in a plane-wave basis is

$$
\begin{aligned}
\hat{H}_{\mathrm{tc}}= & \hat{H}+\frac{1}{2} \sum_{\sigma \sigma^{\prime}} \sum_{p q r s} \omega_{p q}^{r s} a_{p, \sigma}^{\dagger} a_{q, \sigma^{\prime}}^{\dagger} a_{s, \sigma^{\prime}} a_{r, \sigma} \\
& +\frac{1}{2} \sum_{\sigma \sigma^{\prime} \sigma^{\prime \prime}} \sum_{p q o r s t} \omega_{p q o}^{r s t} a_{p, \sigma}^{\dagger} a_{q, \sigma^{\prime}}^{\dagger} a_{o, \sigma^{\prime \prime}}^{\dagger} a_{t, \sigma^{\prime \prime}} a_{s, \sigma^{\prime}} a_{r, \sigma},
\end{aligned}
$$

where momentum conservation requires $\mathbf{k} \equiv \mathbf{k}_{r}-\mathbf{k}_{p}, \mathbf{k}^{\prime} \equiv$ $\mathbf{k}_{q}-\mathbf{k}_{s}$, and $\mathbf{k}_{o}=\mathbf{k}_{t}+\mathbf{k}-\mathbf{k}^{\prime}$, and we define

$$
\begin{aligned}
\omega_{p q}^{r s}= & \frac{1}{\Omega}\left[k^{2} \tilde{u}(\mathbf{k})-\left(\mathbf{k}_{r}-\mathbf{k}_{s}\right) \cdot \mathbf{k} \tilde{u}(\mathbf{k})\right] \\
& +\frac{1}{\Omega} \sum_{\mathbf{k}^{\prime}}\left(\mathbf{k}-\mathbf{k}^{\prime}\right) \cdot \mathbf{k}^{\prime} \tilde{u}\left(\mathbf{k}-\mathbf{k}^{\prime}\right) \tilde{u}\left(\mathbf{k}^{\prime}\right), \\
\omega_{p q o}^{r s t}= & -\frac{1}{\Omega^{2}} \tilde{u}(\mathbf{k}) \tilde{u}\left(\mathbf{k}^{\prime}\right) \mathbf{k} \cdot \mathbf{k}^{\prime} .
\end{aligned}
$$

The TC Hamiltonian has additional two-body and three-body interactions. Due to one of the additional two-body interactions, the TC Hamiltonian is non-Hermitian. This fact can pose some difficulties for variational methods, but not so for projection methods such as full configuration interaction Monte Carlo (FCIQMC) $[9,63]$ and CC.

\section{Approximations to the three-body operator}

The additional three-body operator when treated without approximations will increase the computational scaling of CCD or DCD from $N^{6}$ to $N^{7}$. To seek a good balance between the computational cost and the accuracy, we include only up to effective two-body operators arising from normal-ordering the three-body operator. In this approximation, only the normalordered three-body operator is excluded. We can justify this approximation by analogy to the HF approximation, which constructs a mean-field solution by including only the single and double contractions from the Coulomb operator. In cases where the mean-field approximation is reasonably good, the contribution of the missing normal-ordered Coulomb operator is small, compared with the single and double contractions. In contrast to the HF approximation, the parameters in the correlator in general allow a tuning of the strength of the missing normal-ordered three-body operator, which we will discuss in the next section.

In general, we can write our approximated Hamiltonian as

$$
\begin{aligned}
\hat{H}_{\mathrm{tc}}= & \tilde{E}_{\mathrm{HF}}+\sum_{\sigma} \sum_{p} \tilde{\epsilon}_{p}\left\{a_{p, \sigma}^{\dagger} a_{p, \sigma}\right\} \\
& +\frac{1}{2} \sum_{\sigma \sigma^{\prime}} \sum_{p q r s} \tilde{V}_{p q}^{r s}\left\{a_{p, \sigma}^{\dagger} a_{q, \sigma^{\prime}}^{\dagger} a_{s, \sigma^{\prime}} a_{r, \sigma}\right\} \\
& +E_{\mathrm{T}}+\sum_{\sigma} \sum_{p} \tilde{\omega}_{p}\left\{a_{p, \sigma}^{\dagger} a_{p, \sigma}\right\} \\
& +\frac{1}{2} \sum_{\sigma \sigma^{\prime}} \sum_{p q r s} \tilde{w}_{p q}^{r s}\left\{a_{p, \sigma}^{\dagger} a_{q, \sigma^{\prime}}^{\dagger} a_{s, \sigma^{\prime}} a_{r, \sigma}\right\},
\end{aligned}
$$

where $E_{T}$ refers to the triply contracted three-body operator contribution, $\tilde{\omega}_{p}$ is the doubly contracted three-body integral, and $\tilde{\omega}_{p q}^{r s}$ is the singly contracted three-body integral. The curly brackets indicate that the operators are normal-ordered with respect to the HF vacuum (Fermi sphere). We emphasize that in Eq. (22), $\tilde{E}_{\mathrm{HF}}$ and $\tilde{\epsilon}_{p}$ are calculated now with the modified two-body integrals $\tilde{V}_{p q}^{r s}=w_{p q}^{r s}+V_{p q}^{r s}$. For clarity, we outline the procedures for our TC-CCD/DCD framework.

(1) Evaluating $\omega_{p q}^{r s}$ and $V_{p q}^{r s}$ and combining them into $\tilde{V}_{p q}^{r s} \leftarrow w_{p q}^{r s}+V_{p q}^{r s}$.

(2) Calculating $\tilde{\epsilon}_{p}=\frac{\mathbf{k}_{p}^{2}}{2}+\sum_{i}\left(2 \tilde{V}_{p i}^{p i}-\tilde{V}_{i p}^{p i}\right)$. 


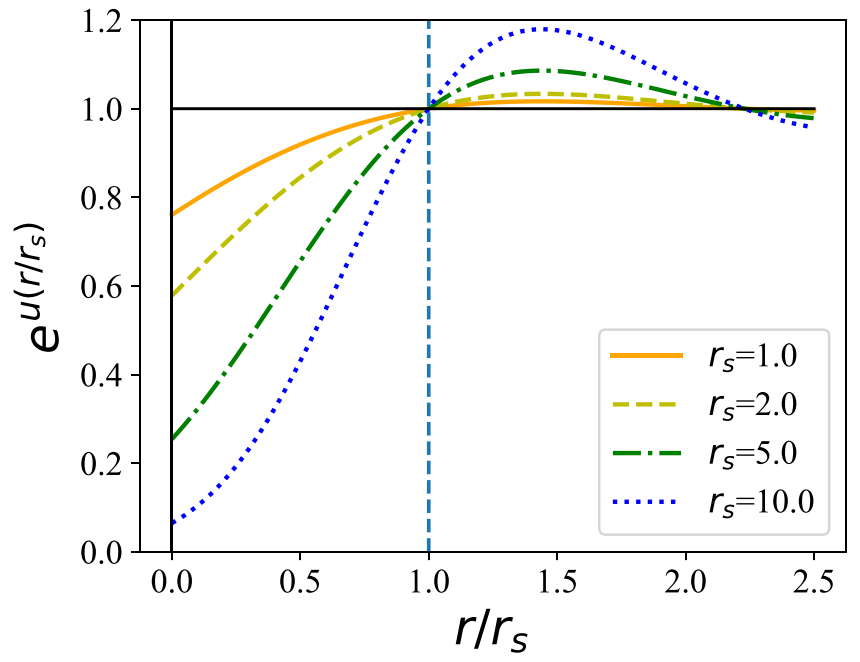

FIG. 1. A sketch of the Jastrow factor with the proposed correlator as the exponent. $E_{\mathrm{T}}$.

(3) Calculating $\tilde{E}_{\mathrm{HF}}=2 \sum_{i}^{N / 2} \tilde{\epsilon}_{i}-\sum_{i j}\left(2 \tilde{V}_{i j}^{i j}-\tilde{V}_{j i}^{i j}\right)$ and

(4) Evaluating $\tilde{\omega}_{p}$, and defining $\epsilon_{p} \leftarrow \tilde{\epsilon}_{p}+\tilde{\omega}_{p}$.

(5) Evaluating the singly contracted three-body integral $\tilde{w}_{p q}^{r s}$ and redefining $V_{p q}^{r s} \leftarrow \tilde{V}_{p q}^{r s}+\tilde{w}_{p q}^{r s}$.

(6) Solving the usual CCD/DCD amplitude equations using $\epsilon_{p}$ and $V_{p q}^{r s}$ for $T_{2}$ and obtaining $E_{\mathrm{c}}$.

(7) The total energy is $E=\tilde{E}_{\mathrm{HF}}+E_{\mathrm{T}}+E_{\mathrm{c}}$.

For details on the mathematical expressions for the contractions of the three-body operator, we refer to the Appendix.

\section{CHOICE AND OPTIMIZATION OF THE CORRELATOR}

Past experience with the TC method has shown that the form of the correlator $\hat{\tau}$ is of extreme importance in the TC method; otherwise, the benefit of TC is lost $-\Phi$ can be simpler than $\Psi$ only if the correlator captures the correct physics of the pair correlations in the system. An inappropriate correlator can actually lead to a harder problem than the original Schrödinger equation. In our previous study of the exact TC method in the UEG, we proposed a form of the correlator (shown below) which was found to work successfully in accelerating convergence to the basis set limit, without changing the correlation that could be captured with the basis set by a FCI level $\Phi$ function. In the present study, since we will be approximating the $\Phi$ with the CC Ansätz, we additionally require the correlator $\hat{\tau}$ to capture some of the correlation inside the Hilbert space.

Here we propose a physically motivated correlator that mimics the behavior of the correlation hole between two unlike-spin electrons as $r_{s}$ varies in 3D UEG. The correlation hole can be examined by the pair-correlation function $g\left(r_{12}\right)$ in real space, as studied in Ref. [64], which shows that the correlation hole between two unlike-spin electrons grows deeper and wider as the Wigner-Seitz radius $r_{s}$ increases or as the electron density decreases. Figure 1 provides a sketch of the Jastrow factor with our proposed correlator $u\left(r_{12}\right)$ as the exponent, which captures the desired behavior. We point out that the functional form of this correlator, which reads in real

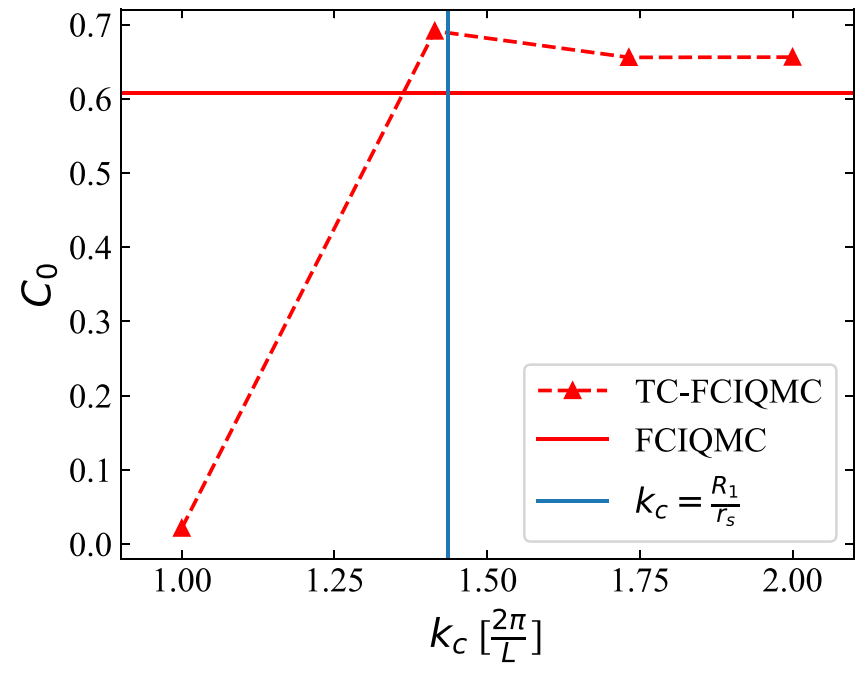

FIG. 2. The weights of the HF determinant (Fermi sphere) as a function of $k_{c}$ extracted from their corresponding TC-FCIQMC simulations (red dashed line) and that of the normal FCIQMC simulation (horizontal red solid line). The vertical blue line is set at $k_{c}=\frac{R_{1}}{r_{r}}$. The system consists of 14 electrons with $r_{s}=5$ and a basis set including 57 plane waves. $5 \times 10^{8}$ walkers are used in all simulations and the initiator threshold is set to 3 . No approximations are made to the three-body interactions.

and reciprocal space, respectively, as

$$
\begin{aligned}
& u(r)=-\frac{r}{\pi}\left(\operatorname{si}\left(k_{c} r\right)+\frac{\cos \left(k_{c} r\right)}{k_{c} r}+\frac{\sin \left(k_{c} r\right)}{\left(k_{c} r\right)^{2}}\right), \\
& \tilde{u}(\mathbf{k})= \begin{cases}-\frac{4 \pi}{k^{4}}, & |\mathbf{k}|>k_{c}, \\
0, & |\mathbf{k}| \leqslant k_{c},\end{cases}
\end{aligned}
$$

where $\operatorname{si}(x)=-\int_{x}^{\infty} \frac{\sin (t)}{t} d t$, was first reported in Ref. [40] to satisfy the cusp condition between two electrons with opposite spins at short interelectron distance and its influence is reduced to nonexistence as the complete basis set (CBS) limit is reached. This was done by choosing $k_{c}$ to be the same as the plane-wave cutoff momentum, $k_{\mathrm{F}}$, which defines how many plane waves are included as basis functions. In contrast, to mimic the behavior of the pair-correlation function, as a first attempt in the present study we choose the parameter in this correlator such that the first nonzero root of Eq. (23) is fixed to be at $r_{s}$, irrespective of the basis set. This is achieved by setting

$$
k_{c}=\frac{R_{1}}{r_{s}},
$$

where $R_{1} \approx 2.322502989$. This choice can be rationalized by the physical picture that at lower densities, electrons prefer to stay further away from each other. Furthermore, this correlator, regardless of the choice of $k_{c}$, retains the cusp condition for two electrons with unlike-spins at $r=0$ [40] and should increase the convergence rate of the computed energies with respect to the employed basis set towards the CBS limit.

To further justify the choice of this correlator, we show that for UEG with 14 electrons at $r_{s}=5$, where traditional CCD exhibits a large error, the most compact expansion of the wave function in Slater determinant space is reached at this value of $k_{c}$. In Fig. 2, we show the weights of the HF determinant 


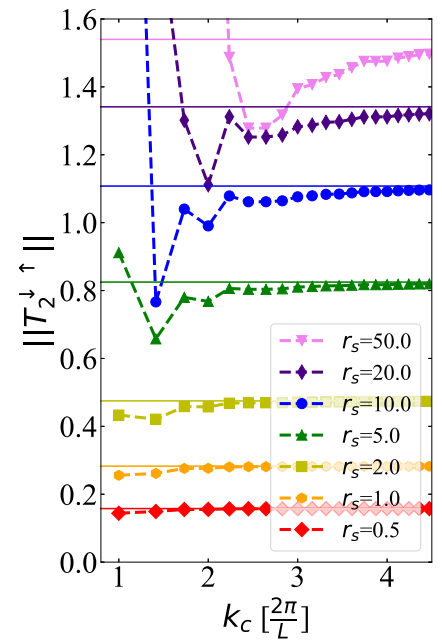

(a)

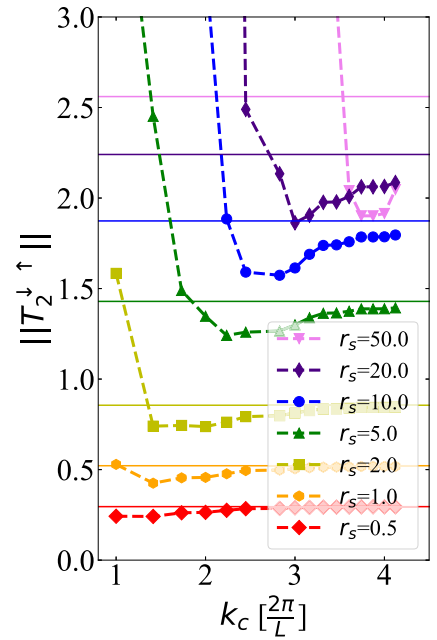

(b)
FIG. 3. The norm of the amplitudes for excitations of unlike-spin electron pairs, $\left\|T_{2}^{\downarrow \uparrow}\right\|$, as a function of $k_{c}$, calculated by the TC-DCD method for (a) the 14-electron and (b) the 54-electron systems. The 14-electron and 54-electron systems use a basis set including 57 and 257 plane waves, respectively. All possible contractions from the three-body interactions are included, excluding the normal-ordered three-body interactions. The solid horizontal color lines, each of which is associated with a $r_{s}$ value indicated by the legend in the same order, represent the $\left\|T_{2}^{\downarrow \uparrow}\right\|$ in the canonical DCD calculations with the same settings as their TC counterparts.

extracted from TC-FCIQMC simulations using different $k_{c}$ values, without making approximations to the three-body operator. We note that due to the discrete momentum mesh as a result of using a finite simulation cell, the possible choices are $k_{c}=\frac{2 \pi \sqrt{n}}{L}, n \in \mathbb{N}$ [65], where $L$ is the length of the cubic cell. In this case, $k_{c}=\frac{R_{1}}{r_{s}}$ is equivalent to $k_{c}=\frac{2 \sqrt{2} \pi}{L}$, and for this choice of $k_{c}$ the exact ground-state wave function of the TC Hamiltonian has the highest weight on the HF determinant.

However, we find that this intuitive choice of $k_{c}$ is not always the optimal, especially at extremely low-density regimes. It is reasonable to expect that the optimal $k_{c}$ for those systems should deviate slightly from $\frac{R_{1}}{r_{s}}$. So we scan a range of $k_{c}$ values around it to locate the one that minimizes the norm of the closed-shell amplitudes for double excitations of electrons with opposite spins, $\left\|T_{2}^{\downarrow \uparrow}\right\|$, in the TC-CCD/DCD calculations with a small basis set, see Fig. 3 [66].

Ideally, two separate correlators should be used for electrons with parallel and anti-parallel spins, and their parameters should be optimized simultaneously using the norm of the full amplitudes in a similar manner. For the present study, we argue that the correlations between two parallel-spin electrons are dominated by the exchange effects, which are already captured by the anti-symmetry in the Slater determinants. Therefore, we focus on capturing the correct physics between electrons with opposite spins in the correlator, i.e., the changing depth and width of the correlation hole as a function of $r_{s}$ [64], and minimizing the corresponding amplitudes in the CC Ansätze. Indeed, we found in practice the minima in $\left\|T_{2}^{\downarrow \uparrow}\right\|$ as a function of $k_{c}$ are more pronounced and thus easier to

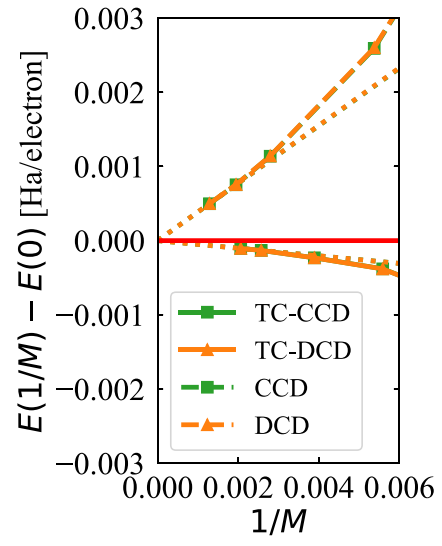

(a)

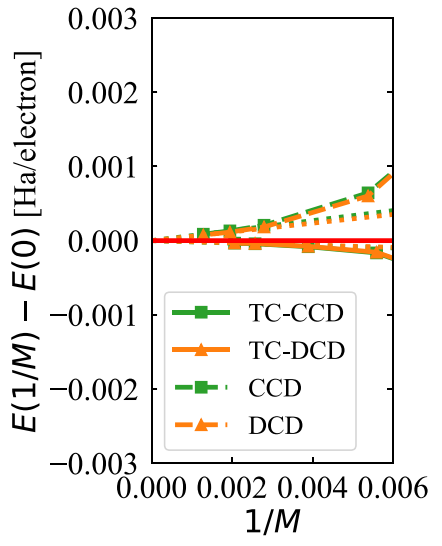

(b)
FIG. 4. Comparison of the basis set convergence behaviors between the canonical CCD/DCD and the TC-CCD/DCD methods. Shown in (a) and (b) is the total energy per electron relative to the extrapolated value retrieved as a function of $1 / M$ at $r_{s}=0.5$ and $r_{s}=5.0$, respectively, where $M$ is the number of plane-wave basis functions for 3D UEG with 14 electrons. Linear extrapolations to the CBS limit using the two leftmost points are used in all cases, shown as the dotted straight lines.

spot than those in the norm of the full amplitudes, $\left\|T_{2}\right\|$. We stress that this compact form of wave function at the optimal choice of $k_{c}$ should greatly benefit approximate methods like CCD and DCD, whose accuracy relies on the assumption that the true ground state wave function is compact around the reference determinant, which is normally chosen to be the HF determinant.

By including the most important contractions, the error of neglecting the rest of the three-body interactions are well under control in that it scales approximately as $\tilde{u}^{2}(k) k^{2} \sim \frac{1}{k^{6}}$. We note in passing that if we choose correlators that do not truncate at small $k$, such as the Yukawa-Coulomb correlator in Ref. [67] or the Gaskell correlator in Ref. [68], the iterative solution of the amplitude equations becomes too unstable to converge at low densities. We attribute this instability in these cases to the large missing normal-ordered three-body interactions, similar to the instability in a $\mathrm{HF}$ self-consistent solution when the missing normal-ordered Coulomb interactions are large.

The FCIQMC calculations are carried out using the NECI program [69]. The CCD and DCD along with the TC integrals are implemented in a Python program using the automatic tensor contraction engine CTF [70] and the NumPy package [71].

\section{RESULTS ON THE 3D UEG}

We first examine the basis set convergence behavior of TC-CCD/DCD compared to the canonical ones. In Fig. 4 we present the total energy per electron relative to the extrapolated value for each method, retrieved as a function of the inverse of the employed number of plane waves, $1 / M$. We use the two leftmost points for linear extrapolations at all densities, since they exhibit consistent linear behavior with the third leftmost data points and the energy differences 
TABLE I. Total energy (Ha/electron), including the Madelung constant, of the 14-electron 3D UEG using different methods. The TCFCIQMC data are from Ref. [40] and the BF-DMC data are from Ref. [54] .

\begin{tabular}{lccccccc}
\hline \hline$r_{s}$ & $k_{c}\left(\frac{2 \pi}{L}\right)$ & CCD & DCD & TC-CCD & TC-DCD & TC-FCIQMC & BF-DMC \\
\hline 0.5 & 1 & 3.41278 & 3.41252 & 3.41258 & 3.41244 & $3.41241(1)$ & $3.41370(2)$ \\
1.0 & 1 & 0.56975 & 0.56909 & 0.56891 & 0.56859 & $0.56861(1)$ & $0.56958(1)$ \\
2.0 & $\sqrt{2}$ & -0.00623 & -0.00748 & -0.00707 & -0.00800 & $-0.00868(2)$ & $-0.007949(7)$ \\
5.0 & $\sqrt{2}$ & -0.07618 & -0.07788 & -0.07816 & -0.07929 & $-0.08002(2)$ & $-0.079706(3)$ \\
10.0 & $\sqrt{2}$ & -0.05137 & -0.05289 & -0.05420 & -0.05509 & N/A & $-0.055160(2)$ \\
20.0 & 2 & -0.02924 & -0.03035 & -0.03136 & -0.03201 & N/A & $-0.0324370(8)$ \\
50.0 & $\sqrt{6}$ & -0.01261 & -0.01323 & -0.01350 & -0.01384 & N/A & $-0.0146251(3)$ \\
\hline \hline
\end{tabular}

${ }^{a}$ The BF-DMC data for the 14-electron 3D UEG are obtained via private communication with P. López Ríos in both this work and Ref. [54]. They were used to produce Fig. 1 in Ref. [54]. However, the values of the data are presented in this work for the first time.

between them are smaller than the targeted accuracy of 0.001 $\mathrm{Ha}$ /electron. As mentioned before, our correlator satisfies the cusp condition at the coalescence point of two electrons with opposite spins. So the accelerated convergence behavior in the TC methods compared with the canonical ones is not surprising. The acceleration is the most obvious at high-density regimes, since at low densities the required number of basis functions to reach convergence in both the TC and non-TC methods is relatively small. These observations are consistent with those of the TC-FCIQMC reported in Ref. [40].

The optimal $k_{c}$ values, (TC-)CCD/DCD energies at CBS and the benchmark data are listed in Tables I and II for the 14and 54-electron 3D UEG, respectively. In Fig. 5 we present the errors of total energies per electron calculated by $\mathrm{TC}$ CCD, TC-DCD, CCD, and DCD relative to the most accurate FCIQMC [40,53,56] and backflow DMC (BF-DMC) [54,72] results on the 14- and 54-electron 3D UEG. The finite basis set errors in our methods have been carefully eliminated by extrapolation to the CBS limit.

In general the accuracy of the TC methods is greatly improved compared with their canonical counterparts, especially in regions $\left(r_{s}=5-50\right)$ where the latter exhibits the largest errors. More importantly, the improved accuracy is retained when going from the 14- to the 54-electron system. We highlight that the TC-DCD achieves an accuracy of $\leqslant 0.001 \mathrm{Ha}$ /electron across a wide range of densities, i.e., $r_{s}=0.5-50$ for the 14-electron and $r_{s}=0.5-20$ for the 54-electron 3D UEG, with an exception at $r_{s}=10$ for the latter where it drops slightly out of the $0.001 \mathrm{Ha} /$ electron accuracy. We argue that with the next possible smaller value of $k_{c}=\sqrt{6}$, which yields a marginally higher $\left\|T_{2}^{\downarrow \uparrow}\right\|$, instead of the current choice of $k_{c}=2 \sqrt{2}$, the $0.001 \mathrm{Ha} /$ electron accuracy at $r_{s}=10$ can be regained. The discrete grid of the $k$-mesh makes it hard to pick the optimal $k_{c}$ in Fig. 3(b). However, as the system gets larger and the $k$-mesh gets finer, the $\left\|T_{2}^{\downarrow \uparrow}\right\|$ as a function of $k_{c}$ will also be smoother, and the choice of the optimal $k_{c}$ will become more definite. We use colorful shaded areas in Fig. 5 to reflect the uncertainties due to the possible choices of $k_{c}$ which yield similar $\left\|T_{2}^{\downarrow \uparrow}\right\|$ values in Fig. 3.

At high densities, i.e., $r_{s}=0.5-2$, the canonical DCD is already very accurate, and the main benefit from TC there is in accelerating the basis set convergence. Overall, DCD exhibits smaller errors than CCD, which agrees with earlier comparative studies between DCSD and CCSD [25-27].

\section{v. CONCLUSIONS}

We demonstrated that the correlator Eq. (23), used with TC-CC theory, drastically improves the accuracy of approximate methods, i.e., CCD and DCD, for 3D UEG across a wide range of densities. The basis set convergence rate is also improved thanks to the fact that the correlator satisfies the cusp condition at the coalescence point of two unlikespin electrons. We have explored the mechanism behind the improved accuracy of the TC-CCD and TC-DCD methods, which is related to a compactification of the many-electron wave function in Slater determinant space when the dominant pair correlations between electrons with unlike-spins are

TABLE II. Total energy (Ha/electron), including the Madelung constant, of the 54-electron 3D UEG using different methods. TC-FCIQMC data are from Ref. [40] and the BF-DMC data are from Ref. [72].

\begin{tabular}{lccccccc}
\hline \hline$r_{s}$ & $k_{c}\left(\frac{2 \pi}{L}\right)$ & CCD & DCD & TC-CCD & TC-DCD & TC-FCIQMC & BF-DMC \\
\hline 0.5 & $\sqrt{2}$ & 3.22079 & 3.22052 & 3.22077 & 3.22071 & $3.22042(2)$ & $3.22112(4)$ \\
1.0 & $\sqrt{2}$ & 0.53069 & 0.53001 & 0.52982 & 0.52968 & $0.52973(3)$ & $0.52989(4)$ \\
2.0 & 2 & -0.01162 & -0.01286 & -0.01324 & -0.01379 & N/A & $-0.01311(2)$ \\
5.0 & $\sqrt{5}$ & -0.07492 & -0.07655 & -0.07750 & -0.07837 & N/A & $-0.079036(3)$ \\
10.0 & $2 \sqrt{2}$ & -0.05016 & -0.05157 & -0.05230 & -0.05322 & N/A & $-0.054443(2)$ \\
20.0 & 3 & -0.02846 & -0.02925 & -0.03055 & -0.03113 & N/A & N/A \\
50.0 & 4 & -0.01223 & -0.01267 & -0.01263 & -0.01281 & N/A \\
\hline \hline
\end{tabular}




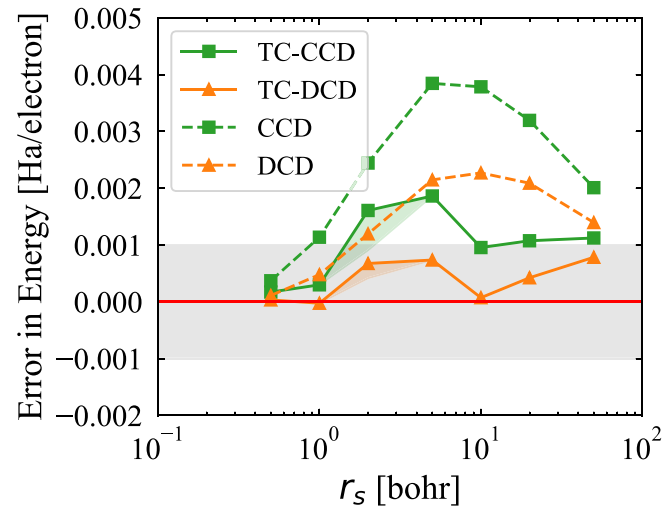

(a)

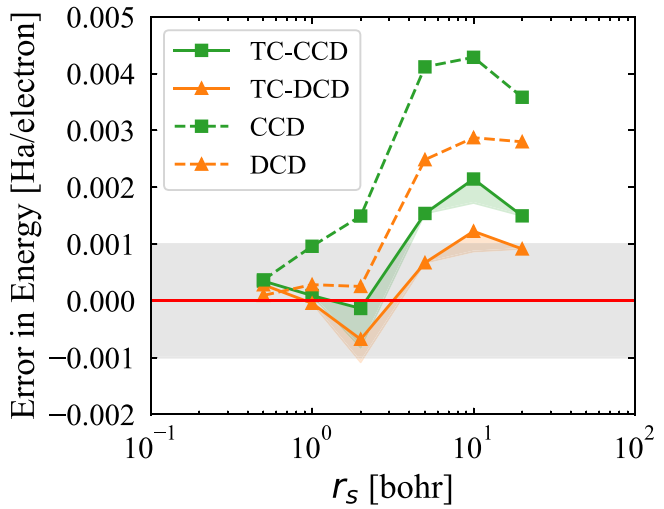

(b)

FIG. 5. Errors in energy per electron relative to benchmark data on the 3D UEG using TC-CCD, TC-DCD, CCD, and DCD methods. (a) Results for 14 electrons. For $r_{s}=0.5-5$, TC-FCIQMC data [40] are used and for the rest BF-DMC data [54] are used for benchmark. (b) Results for 54 electrons. For $r_{s}=0.5-1$, TC-FCIQMC data [40] are used and for the rest BF-DMC data [72] are used for benchmark. The horizontal gray shaded areas stand for the $\pm 0.001 \mathrm{Ha}$ /electron accuracy relative to the reference data. The colorful shaded areas around the data lines reflect the uncertainties in the TC-CCD and TC-DCD energies due to slightly different choices of the $k_{c}$ values.

directly included in the correlator. The optimization of the parameter in the correlator is seamlessly incorporated within the TC-CCD/DCD framework, without requiring an external algorithm. We notice that a range-separation scheme of CCD can also achieve similar accuracy in 3D UEG, but without improving the basis set convergence rate [54]. Comparatively speaking, our methods are systematically improvable, in that a more flexible form of the correlator can be designed by a combination of a series of functions [43] or by a general function approximator, e.g., an artificial neural network, to include further correlation effects such as nucleus-electron correlations and correlations between two parallel-spin electrons in more complicated systems. Other systematic ways of optimizing the correlator in combination with VMC [43] can also be explored. When going to real periodic solids, TC-CCSD and TC-DCSD will be needed; extra efforts are also required to compute the additional integrals besides the Coulomb integrals, where the most computationally demanding part is the singly contracted three-body integrals which scales like $\mathcal{O}\left(N_{o}^{2} N_{v}^{4}\right)$, where $N_{o}$ and $N_{v}$ are the number of occupied and unoccupied orbitals, respectively. Fortunately, the computation of the extra integrals scales no worse than the CCSD or DCSD algorithm and it can be compensated by the accelerated convergence rate towards CBS limit in the TC framework. These perspectives will be important in extending the encouraging performance of the current TC-CCD and TC-DCD methods from the UEG to real periodic solids with moderate to strong correlation.

\section{ACKNOWLEDGMENTS}

We thank A. Grüneis and N. Masios for helpful discussions and P. López Ríos for providing the BF-DMC data for the 14 electron UEG. The authors gratefully acknowledge funding from the Max Planck Society.

The authors declare that they have no competing financial interests.

\section{APPENDIX: INTERNAL CONTRACTIONS IN THE THREE-BODY INTEGRALS}

The three-body integrals can be written as an asymmetric form

$$
\begin{aligned}
\hat{W}_{3}= & -\frac{1}{2 \Omega^{2}} \sum_{\sigma \sigma^{\prime} \sigma^{\prime \prime}} \sum_{\mathbf{k} \mathbf{k}^{\prime} \mathbf{r s t}} \tilde{u}\left(\mathbf{k}^{\prime}\right) \tilde{u}(\mathbf{k}) \mathbf{k}^{\prime} \cdot \mathbf{k} \\
& \times a_{\mathbf{r}-\mathbf{k}, \sigma^{\prime}}^{\dagger} a_{\mathbf{s}+\mathbf{k}^{\prime}, \sigma^{\prime}} a_{\mathbf{t}+\mathbf{k}-\mathbf{k}^{\prime}, \sigma^{\prime \prime}}^{\dagger} a_{\mathbf{t}, \sigma^{\prime \prime}} a_{\mathbf{s}, \sigma^{\prime}} a_{\mathbf{r}, \sigma},
\end{aligned}
$$

where the indices are defined in the main text and conservation of momentum is used. In the following subsections, we will show the specific mathematical expressions for all contractions. The expressions are derived by using the Goldstone diagrams (not shown).

\section{Single contractions}

There are four types of different single contractions, which are

$$
\begin{aligned}
\hat{W}_{3}^{S 1}= & -\frac{N_{e}}{2 \Omega^{2}} \sum_{\sigma \sigma^{\prime}} \sum_{\mathbf{k r s}} \tilde{u}(\mathbf{k})^{2} k^{2}\left\{a_{\mathbf{r}-\mathbf{k}, \sigma}^{\dagger} a_{\mathbf{s}+\mathbf{k}, \sigma^{\prime}}^{\dagger} a_{\mathbf{s}, \sigma^{\prime}} a_{\mathbf{r}, \sigma}\right\}, \\
\hat{W}_{3}^{S 2}= & \frac{1}{\Omega^{2}} \sum_{\sigma \sigma^{\prime}} \sum_{\mathbf{k r s}}\left\{a_{\mathbf{r}-\mathbf{k}, \sigma^{\prime}}^{\dagger} a_{\mathbf{s}+\mathbf{k}, \sigma^{\prime}}^{\dagger} a_{\mathbf{s}, \sigma^{\prime}} a_{\mathbf{r}, \sigma}\right\} \\
& \times \sum_{\mathbf{i}}(\mathbf{i}-\mathbf{r}+\mathbf{k}) \cdot \mathbf{k} \tilde{u}(\mathbf{k}) \tilde{u}(\mathbf{i}-\mathbf{r}+\mathbf{k}), \\
\hat{W}_{3}^{S 3}= & \frac{1}{\Omega^{2}} \sum_{\sigma \sigma^{\prime}} \sum_{\mathbf{k r s}}\left\{a_{\mathbf{r}-\mathbf{k}, \sigma}^{\dagger} a_{\mathbf{s}+\mathbf{k}, \sigma^{\prime}}^{\dagger} a_{\mathbf{s}, \sigma^{\prime}} a_{\mathbf{r}, \sigma}\right\} \\
& \times \sum_{\mathbf{i}}(\mathbf{r}-\mathbf{i}) \cdot \mathbf{k} \tilde{u}(\mathbf{k}) \tilde{u}(\mathbf{r}-\mathbf{i}),
\end{aligned}
$$




$$
\begin{aligned}
\hat{W}_{3}^{S 4}= & \frac{1}{\Omega^{2}} \sum_{\sigma \sigma^{\prime}} \sum_{\mathbf{k r s}}\left\{a_{\mathbf{r}-\mathbf{k}, \sigma}^{\dagger} a_{\mathbf{s}+\mathbf{k}, \sigma^{\prime}}^{\dagger} a_{\mathbf{s}, \sigma^{\prime}} a_{\mathbf{r}, \sigma}\right\} \\
& \times \sum_{\mathbf{i}}(\mathbf{r}-\mathbf{i}-\mathbf{k}) \cdot(\mathbf{r}-\mathbf{i}) \tilde{u}(\mathbf{r}-\mathbf{i}) \tilde{u}(\mathbf{r}-\mathbf{i}-\mathbf{k}),
\end{aligned}
$$

where the curly brackets refer to normal-ordering with respect to the reference determinant. Now we can define the $\tilde{\omega}_{p q}^{r s}$ by the following relation

$$
\begin{array}{r}
\frac{1}{2} \sum_{\sigma \sigma^{\prime \prime}} \sum_{p q r s} \tilde{\omega}_{p q}^{r s}\left\{a_{\mathbf{p}, \sigma}^{\dagger} a_{\mathbf{q}, \sigma^{\prime}}^{\dagger} a_{\mathbf{s}, \sigma^{\prime}} a_{\mathbf{r}, \sigma}\right\} \\
\quad=\hat{W}_{3}^{S 1}+\hat{W}_{3}^{S 2}+\hat{W}_{3}^{S 3}+\hat{W}_{3}^{S 4} .
\end{array}
$$

However, we notice that this effective two-body integral is not symmetric with respect to the exchange of two electrons, due to the fact we used an asymmetric form of the three-body integral. So we need to symmetrize it as follows:

$$
\tilde{\omega}_{p q}^{r s} \leftarrow \frac{1}{2}\left(\tilde{\omega}_{p q}^{r s}+\tilde{\omega}_{q p}^{s r}\right) .
$$

\section{Double contractions}

The double contractions in the three-body integrals result in the $\tilde{\omega}_{p}$ in the main text. It is a sum of five types of double contractions, which reads

$$
\tilde{\omega}_{p}=\frac{N_{e}}{\Omega^{2}}\left(\sum_{\mathbf{i}} \tilde{u}^{2}(\mathbf{p}-\mathbf{i})(\mathbf{p}-\mathbf{i})^{2}\right)
$$

$$
\begin{aligned}
& -\frac{1}{\Omega^{2}}\left(\sum_{\mathbf{i j}}(\mathbf{p}-\mathbf{i}) \cdot(\mathbf{p}-\mathbf{j}) \tilde{u}(\mathbf{p}-\mathbf{i}) \tilde{u}(\mathbf{p}-\mathbf{j})\right) \\
& -\frac{1}{\Omega^{2}}\left(\sum_{\mathbf{i j}}(\mathbf{i}-\mathbf{j}) \cdot(\mathbf{i}-\mathbf{p}) \tilde{u}(\mathbf{i}-\mathbf{j}) \tilde{u}(\mathbf{i}-\mathbf{p})\right) \\
& -\frac{1}{\Omega^{2}}\left(\sum_{\mathbf{i j}}(\mathbf{j}-\mathbf{i}) \cdot(\mathbf{p}-\mathbf{i}) \tilde{u}(\mathbf{j}-\mathbf{i}) \tilde{u}(\mathbf{p}-\mathbf{i})\right) \\
& +\frac{1}{\Omega^{2}}\left(\sum_{\mathbf{i j}}(\mathbf{i}-\mathbf{j})^{2} \tilde{u}^{2}(\mathbf{i}-\mathbf{j})\right) .
\end{aligned}
$$

\section{Triple contractions}

There are two types of triple contractions which contribute to $E_{\mathrm{T}}$ mentioned in the main text:

$$
\begin{aligned}
E_{\mathrm{T}}= & \frac{N_{e}}{2 \Omega^{2}} \sum_{\sigma} \sum_{\mathbf{i j}} \tilde{u}^{2}(\mathbf{i}-\mathbf{j})(\mathbf{i}-\mathbf{j})^{2} \\
& -\frac{1}{\Omega} \sum_{\sigma} \sum_{\mathbf{i j}}\left(\frac{1}{\Omega} \sum_{\mathbf{k}}(\mathbf{i}-\mathbf{k}) \cdot(\mathbf{i}-\mathbf{j}) \tilde{u}(\mathbf{i}-\mathbf{j}) \tilde{u}(\mathbf{i}-\mathbf{k})\right) .
\end{aligned}
$$

[1] J. Čížek, On the correlation problem in atomic and molecular systems. calculation of wavefunction components in ursell-type expansion using quantum-field theoretical methods, J. Chem. Phys. 45, 4256 (1966).

[2] J. Čižek and J. Paldus, Correlation problems in atomic and molecular systems III. Rederivation of the coupled-pair many-electron theory using the traditional quantum chemical methodst, Int. J. Quantum Chem. 5, 359 (1971).

[3] J. Č́̌žek and J. Paldus, Coupled cluster approach, Phys. Scr. 21, 251 (1980).

[4] K. Raghavachari, G. W. Trucks, J. A. Pople, and M. HeadGordon, A fifth-order perturbation comparison of electron correlation theories, Chem. Phys. Lett. 157, 479 (1989).

[5] C. Riplinger, P. Pinski, U. Becker, E. F. Valeev, and F. Neese, Sparse maps-A systematic infrastructure for reduced-scaling electronic structure methods. II. Linear scaling domain based pair natural orbital coupled cluster theory, J. Chem. Phys. 144, 024109 (2016).

[6] G. Schmitz and C. Hättig, Perturbative triples correction for local pair natural orbital based explicitly correlated $\operatorname{CCSD}(\mathrm{F} 12 *)$ using Laplace transformation techniques, J. Chem. Phys. 145, 234107 (2016).

[7] M. Schwilk, Q. Ma, C. Köppl, and H.-J. Werner, Scalable electron correlation methods. 3. efficient and accurate parallel local coupled cluster with pair natural orbitals (PNO-LCCSD), J. Chem. Theory Comput. 13, 3650 (2017).
[8] P. R. Nagy, G. Samu, and M. Kállay, Optimization of the linear-scaling local natural orbital $\operatorname{CCSD}(\mathrm{T})$ method: improved algorithm and benchmark applications, J. Chem. Theory Comput. 14, 4193 (2018).

[9] G. H. Booth, A. Grüneis, G. Kresse, and A. Alavi, Towards an exact description of electronic wavefunctions in real solids, Nature (London) 493, 365 (2013).

[10] K. Liao and A. Grüneis, Communication: Finite size correction in periodic coupled cluster theory calculations of solids, J. Chem. Phys. 145, 141102 (2016).

[11] J. McClain, Q. Sun, G. K.-L. Chan, and T. C. Berkelbach, Gaussian-based coupled-cluster theory for the ground-state and band structure of solids, J. Chem. Theory Comput. 13, 1209 (2017).

[12] T. Gruber, K. Liao, T. Tsatsoulis, F. Hummel, and A. Grüneis, Applying the Coupled-Cluster Ansatz to Solids and Surfaces in the Thermodynamic Limit, Phys. Rev. X 8, 021043 (2018).

[13] K. Liao, X.-Z. Li, A. Alavi, and A. Grüneis, A comparative study using state-of-the-art electronic structure theories on solid hydrogen phases under high pressures, npj Comput. Mater. 5, 110 (2019).

[14] Y. Gao, Q. Sun, J. M. Yu, M. Motta, J. McClain, A. F. White, A. J. Minnich, and Garnet Kin-Lic Chan, Electronic structure of bulk manganese oxide and nickel oxide from coupled cluster theory, Phys. Rev. B 101, 165138 (2020). 
[15] J. J. Shepherd and A. Grüneis, Many-Body Quantum Chemistry for the Electron Gas: Convergent Perturbative Theories, Phys. Rev. Lett. 110, 226401 (2013).

[16] W. Meyer, Ionization energies of water from PNO-CI calculations, Int. J. Quantum Chem. 5, 341 (1971).

[17] M. Nooijen and R. J. Le Roy, Orbital invariant single-reference coupled electron pair approximation with extensive renormalized triples correction, J. Mol. Struct (THEOCHEM), 768, 25 (2006).

[18] F. Neese, F. Wennmohs, and A. Hansen, Efficient and accurate local approximations to coupled-electron pair approaches: An attempt to revive the pair natural orbital method, J. Chem. Phys. 130, 114108 (2009).

[19] L. M. J. Huntington and M. Nooijen, pCCSD: Parameterized coupled-cluster theory with single and double excitations, J. Chem. Phys. 133, 184109 (2010).

[20] J. Paldus, J. Č́žžek, and M. Takahashi, Approximate account of the connected quadruply excited clusters in the coupled-pair many-electron theory, Phys. Rev. A 30, 2193 (1984).

[21] P. Piecuch and J. Paldus, On the solution of coupled-cluster equations in the fully correlated limit of cyclic polyene model, Int. J. Quantum Chem. 40, 9 (1991).

[22] R. J. Bartlett and M. Musiał, Addition by subtraction in coupled-cluster theory: A reconsideration of the CC and CI interface and the nCC hierarchy, J. Chem. Phys. 125, 204105 (2006).

[23] J. B. Robinson and P. J. Knowles, Approximate variational coupled cluster theory, J. Chem. Phys. 135, 044113 (2011).

[24] D. W. Small, K. V. Lawler, and M. Head-Gordon, Coupled cluster valence bond method: efficient computer implementation and application to multiple bond dissociations and strong correlations in the acenes, J. Chem. Theory Comput. 10, 2027 (2014).

[25] D. Kats and F. R. Manby, Communication: The distinguishable cluster approximation, J. Chem. Phys. 139, 021102 (2013).

[26] D. Kats, Communication: The distinguishable cluster approximation. II. The role of orbital relaxation, J. Chem. Phys. 141, 061101 (2014).

[27] D. Kats, D. Kreplin, H. J. Werner, and F. R. Manby, Accurate thermochemistry from explicitly correlated distinguishable cluster approximation, J. Chem. Phys. 142, 064111 (2015).

[28] V. Rishi, A. Perera, M. Nooijen, and R. J. Bartlett, Excited states from modified coupled cluster methods: Are they any better than EOM CCSD?, J. Chem. Phys. 146, 144104 (2017).

[29] T. Tsatsoulis, F. Hummel, D. Usvyat, M. Schütz, G. H. Booth, S. S. Binnie, M. J. Gillan, D. Alfè, A. Michaelides, and A. Grüneis, A comparison between quantum chemistry and quantum Monte Carlo techniques for the adsorption of water on the (001) LiH surface, J. Chem. Phys. 146, 204108 (2017).

[30] S. F. Boys and N. C. Handy, A condition to remove the indeterminacy in interelectronic correlation functions, Proc. R. Soc. Math. Phys. Eng. Sci. 309, 209 (1969).

[31] S. F. Boys and N. C. Handy, The determination of energies and wavefunctions with full electronic correlation, Proc. R. Soc. Math. Phys. Eng. Sci. 310, 43 (1969).

[32] S. F. Boys, Some bilinear convergence characteristics of the solutions of dissymmetric secular equations, Proc. R. Soc. Math. Phys. Eng. Sci. 309, 195 (1969).
[33] O. Hino, Y. Tanimura, and S. Ten-no, Biorthogonal approach for explicitly correlated calculations using the transcorrelated Hamiltonian, J. Chem. Phys. 115, 7865 (2001).

[34] O. Hino, Y. Tanimura, and S. Ten-no, Application of the transcorrelated Hamiltonian to the linearized coupled cluster singles and doubles model, Chem. Phys. Lett. 353, 317 (2002).

[35] N. Umezawa and S. Tsuneyuki, Ground-state correlation energy for the homogeneous electron gas calculated by the transcorrelated method, Phys. Rev. B 69, 165102 (2004).

[36] R. Sakuma and S. Tsuneyuki, Electronic structure calculations of solids with a similarity-transformed hamiltonian, J. Phys. Soc. Jpn. 75, 103705 (2006).

[37] M. Ochi and S. Tsuneyuki, Second-order Møller-Plesset perturbation theory for the transcorrelated Hamiltonian applied to solid-state calculations, Chem. Phys. Lett. 621, 177 (2015).

[38] M. Ochi, Y. Yamamoto, R. Arita, and S. Tsuneyuki, Iterative diagonalization of the non-Hermitian transcorrelated Hamiltonian using a plane-wave basis set: Application to sp-electron systems with deep core states, J. Chem. Phys. 144, 104109 (2016).

[39] M. Ochi, R. Arita, and S. Tsuneyuki, Correlated Band Structure of a Transition Metal Oxide ZnO Obtained from a Many-Body Wave Function Theory, Phys. Rev. Lett. 118, 026402 (2017).

[40] H. Luo and A. Alavi, Combining the transcorrelated method with full configuration interaction quantum Monte Carlo: application to the homogeneous electron gas, J. Chem. Theory Comput. 14, 1403 (2018).

[41] P. Jeszenszki, H. Luo, A. Alavi, and J. Brand, Accelerating the convergence of exact diagonalization with the transcorrelated method: Quantum gas in one dimension with contact interactions, Phys. Rev. A 98, 053627 (2018).

[42] W. Dobrautz, H. Luo, and A. Alavi, Compact numerical solutions to the two-dimensional repulsive Hubbard model obtained via nonunitary similarity transformations, Phys. Rev. B 99, 075119 (2019).

[43] A. J. Cohen, H. Luo, K. Guther, W. Dobrautz, D. P. Tew, and A. Alavi, Similarity transformation of the electronic Schrödinger equation via Jastrow factorization, J. Chem. Phys. 151, 061101 (2019).

[44] P. Jeszenszki, U. Ebling, H. Luo, A. Alavi, and J. Brand, Eliminating the wave-function singularity for ultracold atoms by a similarity transformation, Phys. Rev. Research 2, 043270 (2020).

[45] E. Wigner, On the interaction of electrons in metals, Phys. Rev. 46, 1002 (1934).

[46] D. M. Ceperley and B. J. Alder, Ground State of the Electron Gas by a Stochastic Method, Phys. Rev. Lett. 45, 566 (1980).

[47] W. Kohn and L. J. Sham, Self-consistent equations including exchange and correlation effects, Phys. Rev. 140, A1133 (1965).

[48] J. P. Perdew and A. Zunger, Self-interaction correction to density-functional approximations for many-electron systems, Phys. Rev. B 23, 5048 (1981).

[49] J. P. Perdew, K. Burke, and M. Ernzerhof, Generalized Gradient Approximation Made Simple, Phys. Rev. Lett. 77, 3865 (1996).

[50] D. Bohm and D. Pines, A collective description of electron interactions: III. coulomb interactions in a degenerate electron gas, Phys. Rev. 92, 609 (1953).

[51] M. Gell-Mann and K. A. Brueckner, Correlation energy of an electron gas at high density, Phys. Rev. 106, 364 (1957). 
[52] J. J. Shepherd, G. Booth, A. Grüneis, and A. Alavi, Full configuration interaction perspective on the homogeneous electron gas, Phys. Rev. B 85, 081103(R) (2012).

[53] J. J. Shepherd, G. H. Booth, and A. Alavi, Investigation of the full configuration interaction quantum Monte Carlo method using homogeneous electron gas models, J. Chem. Phys. 136, 244101 (2012).

[54] J. J. Shepherd, T. M. Henderson, and G. E. Scuseria, RangeSeparated Brueckner Coupled Cluster Doubles Theory, Phys. Rev. Lett. 112, 133002 (2014).

[55] J. J. Shepherd, T. M. Henderson, and G. E. Scuseria, Coupled cluster channels in the homogeneous electron gas, J. Chem. Phys. 140, 124102 (2014).

[56] V. A. Neufeld and A. J. W. Thom, A study of the dense uniform electron gas with high orders of coupled cluster, J. Chem. Phys. 147, 194105 (2017)

[57] M. Ruggeri, P. L. Ríos, and A. Alavi, Correlation energies of the high-density spin-polarized electron gas to meV accuracy, Phys. Rev. B 98, 161105(R) (2018).

[58] A. Irmler, A. Gallo, F. Hummel, and A. Grüneis, Duality of Ring and Ladder Diagrams and its Importance for ManyElectron Perturbation Theories, Phys. Rev. Lett. 123, 156401 (2019).

[59] D. Kats and F. R. Manby, Sparse tensor framework for implementation of general local correlation methods, J. Chem. Phys. 138, 144101 (2013).

[60] D. Kats, The distinguishable cluster approach from a screened Coulomb formalism, J. Chem. Phys. 144, 44102 (2016).

[61] P. Pulay, Convergence acceleration of iterative sequences. the case of scf iteration, Chem. Phys. Lett. 73, 393 (1980).

[62] P. Pulay, Improved SCF convergence acceleration, J. Comput. Chem. 3, 556 (1982).

[63] G. H. Booth, A. J. Thom, and A. Alavi, Fermion Monte Carlo without fixed nodes: A game of life, death, and annihilation in Slater determinant space, J. Chem. Phys. 131, 054106 (2009).
[64] P. Gori-Giorgi, F. Sacchetti, and G. B. Bachelet, Analytic structure factors and pair-correlation functions for the unpolarized homogeneous electron gas, Phys. Rev. B 61, 7353 (2000).

[65] $n=n_{1}^{2}+n_{2}^{3}+n_{3}^{2}$, where $n_{1}, n_{2}, n_{3}$ are the components of a $\mathbf{k}$ vector in units of $\frac{2 \pi}{L}$. So the possible values of $n$ are $0,1,2,3$, $4,5,6,8, \ldots$.

[66] Since the $\left\|T_{2}^{\downarrow \uparrow}\right\|$ in TC-CCD and TC-DCD show the same trend as a function of $k_{c}$, we show only the latter in the figure.

[67] A. Grüneis, J. J. Shepherd, A. Alavi, D. P. Tew, and G. H. Booth, Explicitly correlated plane waves: Accelerating convergence in periodic wavefunction expansions, J. Chem. Phys. 139, 084112 (2013).

[68] T. Gaskell, The collective treatment of a fermi gas: II, Proc. Phys. Soc. 77, 1182 (1961).

[69] K. Guther, R. J. Anderson, N. S. Blunt, N. A. Bogdanov, D. Cleland, N. Dattani, W. Dobrautz, K. Ghanem, P. Jeszenszki, N. Liebermann, G. L. Manni, A. Y. Lozovoi, H. Luo, D. Ma, F. Merz, C. Overy, M. Rampp, P. K. Samanta, L. R. Schwarz, J. J. Shepherd et al., NECI: N-electron configuration interaction with an emphasis on state-of-the-art stochastic methods, J. Chem. Phys. 153, 034107 (2020).

[70] E. Solomonik, D. Matthews, J. R. Hammond, J. F. Stanton, and J. Demmel, A massively parallel tensor contraction framework for coupled-cluster computations, J. Parallel Distrib. Comput. 74, 3176 (2014).

[71] C. R. Harris, K. J. Millman, S. J. van der Walt, R. Gommers, P. Virtanen, D. Cournapeau, E. Wieser, J. Taylor, S. Berg, N. J. Smith, R. Kern, M. Picus, S. Hoyer, M. H. van Kerkwijk, M. Brett, A. Haldane, J. F. del Río, M. Wiebe, P. Peterson, P. Gérard-Marchant et al., Array programming with NumPy, Nature (London) 585, 357 (2020).

[72] P. López Ríos, A. Ma, N. D. Drummond, M. D. Towler, and R. J. Needs, Inhomogeneous backflow transformations in quantum Monte Carlo calculations, Phys. Rev. E 74, 066701 (2006). 